\title{
Validation of Synthetic Diamond for a Beam Condition Monitor for the Compact Muon Solenoid Experiment
}

\author{
Daniel Chong, Luis Fernandez-Hernando, Richard Gray, Christoph J. Ilgner, Member, IEEE, Alick L. Macpherson, \\ Alexander Oh, Terry W. Pritchard, Robert Stone, and Steven Worm
}

\begin{abstract}
The CERN Large Hadron Collider (LHC) will collide two counter rotating proton beams. The energy stored in each beam is about $350 \mathrm{MJ}$. If there is a failure in an element of the accelerator, the resulting beam losses could cause damage not only to the accelerator itself, but also to the physics experiments installed. Within the Compact Muon Solenoid experiment (CMS), the use of a Beam Condition Monitor (BCM) is planned to monitor a possible fast increase of the particle flux near the interaction point. The system will flag the onset of adverse beam conditions within the CMS experiment, and, if necessary, input into the beam abort system of the LHC requesting a fast beam dump. Constraints on BCM design from radiation hardness, a minimal material and services budget, and the need for fast signals from sensors with high sensitivity and a large dynamic range, have led to the investigation of synthetic chemical vapor deposited (CVD) diamond for the BCM sensor. In a $5 \mathrm{GeV}$ hadron beam the response of CVD diamonds was tested in fluxes ranging from single minimium ionizing particles (MIPs) to $10^{8}$ MIPs per $\mathrm{cm}^{2}$, delivered over $40 \mathrm{~ns}$. The signal response, linearity, and stability of both un-irradiated and irradiated sensors were tested over this flux range, and will be presented herein. The results confirm the applicability of CVD diamond for this beam monitoring application.
\end{abstract}

Index Terms-Beam abort, chemical vapor deposition diamond, position sensitive detectors, radiation detection: radiation detectors, semiconductor detectors: characterization.

\section{THE BEAM CONDITION MONITOR}

$\mathbf{I}$ $T$ is envisaged that the Beam Condition Monitor (BCM) will provide real-time bunch-by-bunch monitoring of the beam conditions within the Compact Muon Solenoid (CMS) experiment at CERN, and will be used to flag unsuitable beam conditions, the onset of beam instabilities, and beam accidents. The BCM will monitor the $25 \mathrm{~ns}$ inter-bunch spacing of the LHC beam, and give monitoring feedback to the accelerator both in normal running, and during the initial LHC pilot run. It will

Manuscript received November 15, 2004; revised October 25, 2006.

D. Chong is with the University of Canterbury, Christchurch 8140, New Zealand.

L. Fernandez-Hernando is with CERN, CH-1211 Geneva 23, Switzerland and also with EPFL, CH-1015 Lausanne, Switzerland.

R. Gray is with the University of Auckland, Auckland 1142, New Zealand.

C. J. Ilgner and A. Oh are with CERN, CH-1211 Geneva 23, Switzerland (e-mail: Christoph.Ilgner@cern.ch).

A. L. Macpherson is with CERN, CH-1211 Geneva 23, Switzerland and also with Paul-Scherrer-Institut, CH-5232 Villigen, Switzerland.

T. W. Pritchard is with Rutherford Appleton Laboratory, Chilton, Didcot OX11 0QX, U.K.

R. Stone and S. Worm are with Rutgers University, New Brunswick, NJ 08903 USA.

Digital Object Identifier 10.1109/TNS.2006.889644 also form the central component of the CMS radiation monitoring system, being able to initiate actions within the CMS Detector Safety System (DSS) [1] and the CMS Detector Control Systems (DCS). In case of a severe failure of accelerator-control systems, leading to equipment damage [2], its time-stamped monitoring data will be used for later analysis.

The BCM is designed to complement the LHC machine protection system [3], and on detection of abnormal particle fluxes within the CMS detector, will directly input into the LHC beam interlock system. Fast and reliable signals from the BCM are crucial, reflecting the $25 \mathrm{~ns}$ time structure of the LHC beams, as it will form the sole means by which CMS can initiate a fast request to abort the LHC beams.

\section{CVD DIAMOND AS BCM SENSOR MATERIAL}

Due to limited space available inside the CMS experiment and a restricted material budget, resulting from the need to prevent multiple scattering, polycrystalline CVD (chemical vapor deposition) diamond was chosen for investigation as the BCM sensor because of its high radiation hardness [4], fast response time [5], and minimal services requirements. For an application as a BCM, a CVD diamond sensor needs to have an active area of about $1 \mathrm{~cm}^{2}$.

Diamond is an insulator, so the sensitive volume of a CVD diamond detector is given by the geometry of the applied electric field, i.e. the shape of the electrodes. Upon the passage of a charged particle, the secondary free charge carriers produced by ionization processes start to drift under the influence of the electric field. As the charge distribution changes, image charges are induced on the electrodes, which are compensated by the flow of current in the external circuit, which can be detected [5].

The charge collection properties of several diamond samples were characterized with beta particles from a ${ }^{90} \mathrm{Sr}$ source simulating minimum ionizing particles (MIPs). A MIP in diamond generates on average 36 electron-hole pairs per $\mu \mathrm{m}$ [6].

The collection distance $\delta$ is a measure of the efficiency of collecting the liberated charge in the material. According to a definition given in [7], the collection distance $\delta$ equals $\mu \tau E$, with $\mu$ mobility of the charge carriers, $\tau$ : their average life time and $E$ : electric field strength. For large detector thicknesses $(d \gg d)$, the collection distance is

$$
\delta \approx \frac{Q_{C}}{Q_{G}} \cdot d
$$




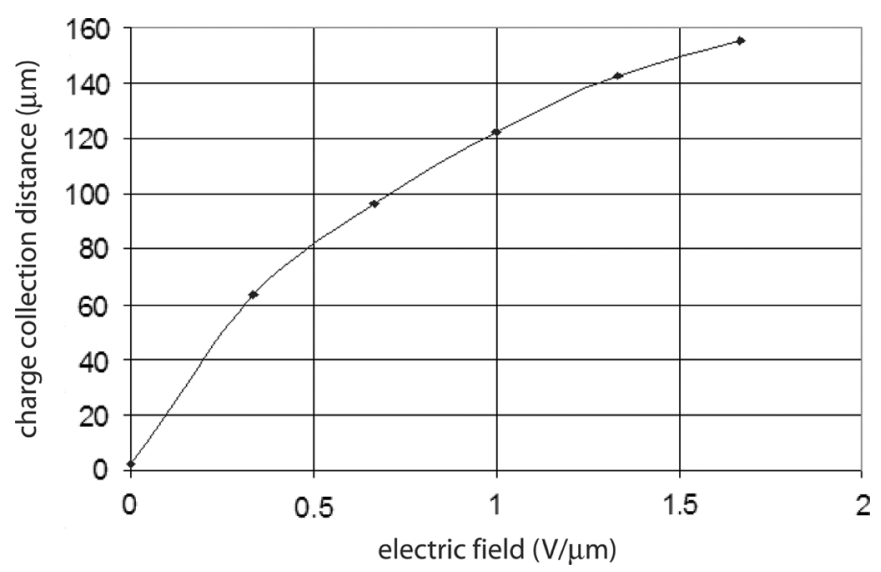

Fig. 1. The dependency of the collection distance $\delta$ for a diamond sample of $300 \mu \mathrm{m}$ thickness on the electric field $\mathrm{E}$.

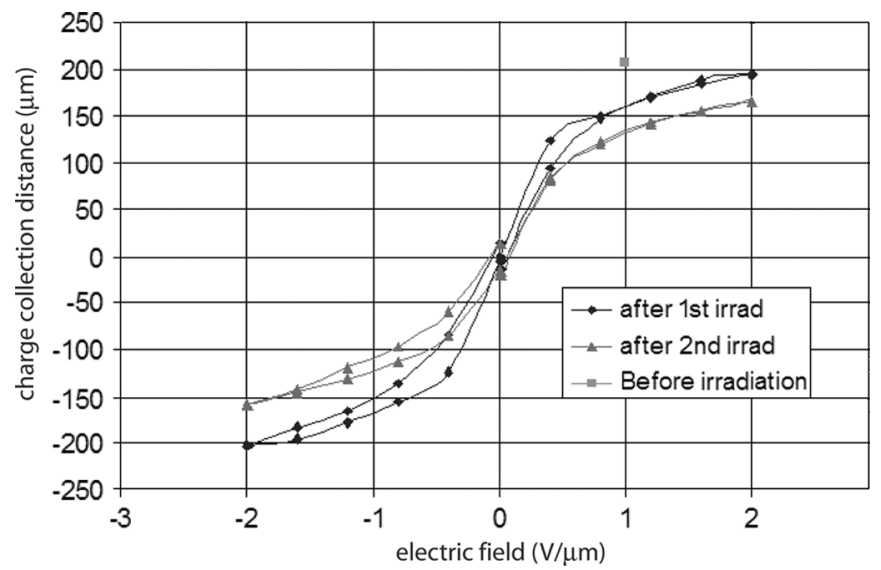

Fig. 2. $\delta$ versus electric field after an irradiation of $10^{15}$ protons per $\mathrm{cm}^{2}$ (first irradiation) and after $2.8 \cdot 10^{15}$ protons per $\mathrm{cm}^{2}$ (second irradiation), in a $500 \mu \mathrm{m}$ thick polycrystalline CVD diamond sensor. The value of $\delta$ before irradiation at $1 \mathrm{~V} / \mu \mathrm{m}$ bias is also given.

where $Q_{C}$ is the collected charge, $Q_{G}$ the primary ionization charge liberated by the ionizing particle and $\mathrm{d}$ the detector thickness. The latter is the definition used here. In the case of small efficiencies, which again can be expressed as $d \gg \delta$, the collection distance corresponds to the sum of the average drift distance of electrons and holes.

Fig. 1 shows the collection distance $\delta$ for a diamond sample of $300 \mu \mathrm{m}$ thickness.

The radiation tolerance of several diamond samples was tested with protons of $24 \mathrm{GeV} / \mathrm{c}$ up to a fluence of $2.8 \cdot 10^{15} / \mathrm{cm}^{2}$ (fluence expected after 10 years of normal operating conditions at the sensor position inside the CMS experiment [8]). Fig. 2 shows that the charge collection distance $\delta$, which is used as a measure for the sensor quality, has only dropped by roughly $30 \%$ after having received that proton fluence.

\section{Response to High Intensity BeAms}

Since in several accident scenarios, like the unsynchronized beam abort [9]-[11], the BCM sensors will be exposed to extremely high particle fluxes and will still need to respond, they were also tested in a radiation environment that approximates these conditions.

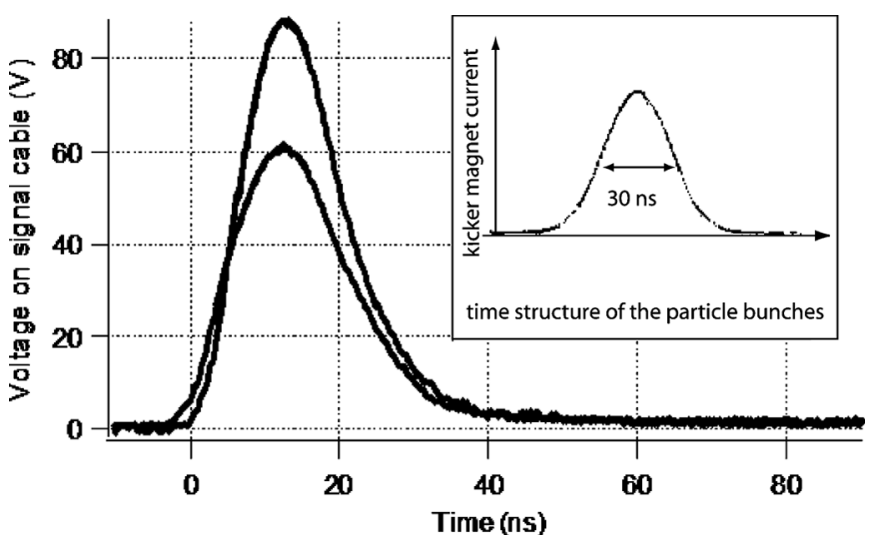

Fig. 3. The bare sensor response (no amplification) into a $50 \mathrm{ohms} \mathrm{load} \mathrm{for} \mathrm{a}$ single bunch from two diamond sensors (500 $\mu \mathrm{m}$ thick, top trace, and $300 \mu \mathrm{m}$ thick, bottom trace), at a position where the total fluence was $(2.2 \pm 0.2)$. $10^{8}$ protons $/ \mathrm{cm}^{2}$. The inset shows the current in the kicker magnet of the accelerator, which extracts the particles from the storage ring. It indicates the time structure of the particle bunch, the so-called spill, as it hits the diamond sensor (halfwidth $=30 \mathrm{~ns}$ ).

TABLE I

CURRENT AMPLITUdeS AT A PARTICLE FLUX OF $9.8 \cdot 10^{7} /\left(\mathrm{cm}^{2} \cdot \mathrm{spill}\right)$ AND 50 ohms LOAD. A SPILl IS ONE EXTRACTION OF PARTICles From THE ACCELERATOR, ITS HALFWIDTH Is 30 ns (SEE FIG. 3)

\begin{tabular}{|l|c|c|}
\hline diamond thickness & $300 \mu \mathrm{m}$ & $500 \mu \mathrm{m}$ \\
\hline current amplitude & $1.22 \mathrm{~A}$ & $1.76 \mathrm{~A}$ \\
\hline
\end{tabular}

The experiments carried out in a $5 \mathrm{GeV}$ hadron beam, delivered over 40 ns by the CERN Proton Synchrotron with fluxes ranging from single minimium ionizing particles (MIPs) to $10^{8}$ MIPs per $\mathrm{cm}^{2}$, show that the signals from CVD sensors are large. Fig. 3 shows two examples of raw signals from diamond sensors of different thicknesses, without amplification, at a bias voltage of $1 \mathrm{~V} / \mu \mathrm{m}$. Current amplitudes at lower particle fluxes are shown in Table I. The diamond sensors were arranged in a way that the incoming particles' trajectories were perpendicular to the metallization.

To assess the diamond-sensor response to consecutive bunches of particles, the T7 beamline at the CERN Proton Synchrotron was set up in a way that it could deliver $40 \mathrm{~ns}$ long beam bunches of approximately $10^{9}$ particles onto the test setup, with consecutive bunches being $262 \mathrm{~ns}$ apart. A consecutive set of bunches is called a spill. The behavior of the tested CVD diamond sensors under these conditions is shown in Fig. 4, together with a pSpice simulation of the bias voltage. Here the spill structure is clearly seen, with no long term baseline effects after a 7-bunch spill with each bunch corresponding to a flux of approximately $10^{8}$ protons $/ \mathrm{cm}^{2}$.

In order to verify the stability of the testbeam size and shape for both single-particle and high-intensity-beam extraction from the CERN Proton Synchrotron, a beam profile was taken. In the single-particle beam scintillators were used, and CVD diamond sensors in the high-intensity beam. Fig. 5 shows an excellent agreement, inferring that the diamond response is proportional to the particle flux. Aluminium dosimetry was used to measure the particle fluences.

For the application of CVD diamond as a BCM sensor, the option of placing the sensor parallel to the beam (see Fig. 6, bottom 


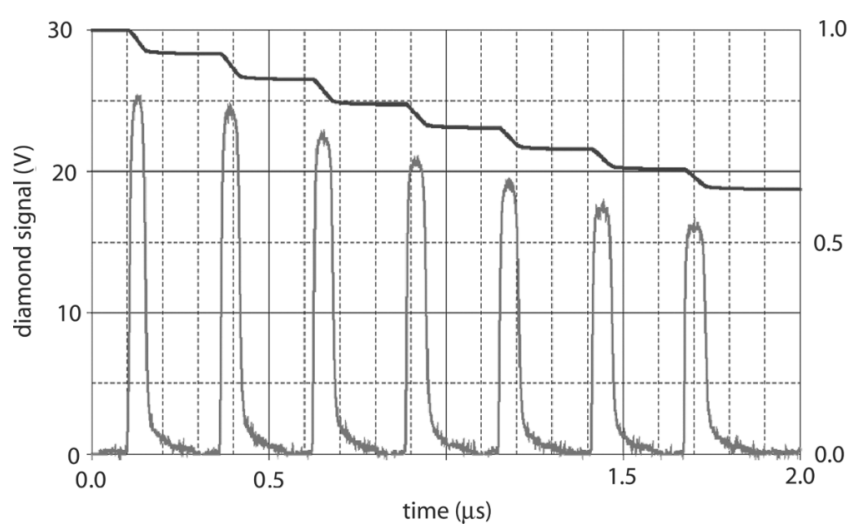

Fig. 4. The grey curve represents the signal from consecutive bunches at $10^{8} \mathrm{p} /\left(\mathrm{cm}^{2}\right.$. spill) into a 50 ohms load. The bunch structure of the beam is clearly visible with the interbunch spacing of $262 \mathrm{~ns}$. The bias field on the diamond sensor after each bunch is also shown (black curve); as the reservoir capacitor discharges with time, the bias field decreases.

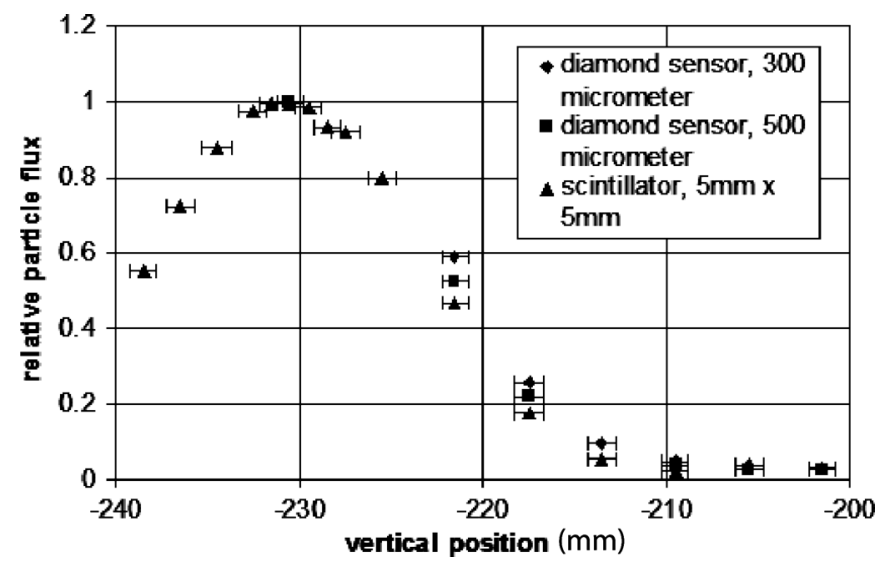

Fig. 5. Beam profile (relative particle flux as a function of the vertical position of the sensors) measured by scintillators (active surface: $5 \mathrm{~mm} \times 5 \mathrm{~mm}$ ) and with CVD diamond sensors during high-intensity irradiation (40 ns spill duration).

scheme) was investigated, which promises to yield higher signals from minimum-ionizing particles, since the particles pass through $10 \mathrm{~mm}$ of sensor material. The amplitude spectrum for this CVD diamond sensor configuration after preamplification (amplifier gain : $15.8=24 \mathrm{~dB}$ ) is given in Fig. 6. This sensor configuration leads to a higher signal from minimum-ionizing particles, but the passage of non-aligned particles leads to a low-end tail in the amplitude spectrum, representing a reduction of the sensor's angular coverage.

In order to set up a system that can provide monitoring information during normal operation as well as fast response to beam accidents, the linearity of the CVD diamond sensor response to particle fluences covering 8 orders of magnitude was also investigated. Fig. 7 shows the response of the sensors which was found to be linear within the range tested.

The particle fluence has been meeasured by a variety of independent means, such as scintillator particle counting, thermoluminescence and aluminium dosimetry. The linearity of the CVD-diamond response allows to use this type of sensors for

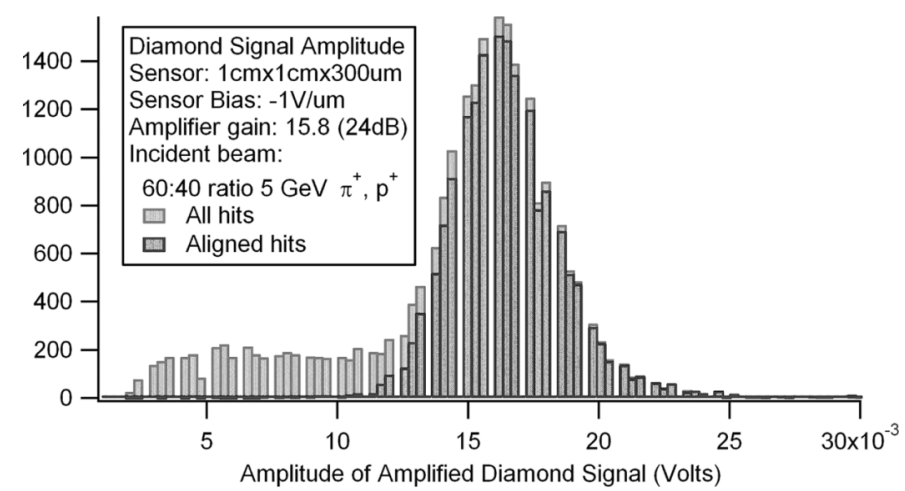

(a)

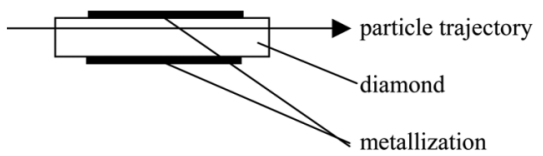

(b)

Fig. 6. Amplitude distribution after preamplification for a diamond sensor oriented parallel to the particle beam (see bottom scheme), i.e. the particles' trajectory is parallel to the metallized areas of the CVD diamond sensor.

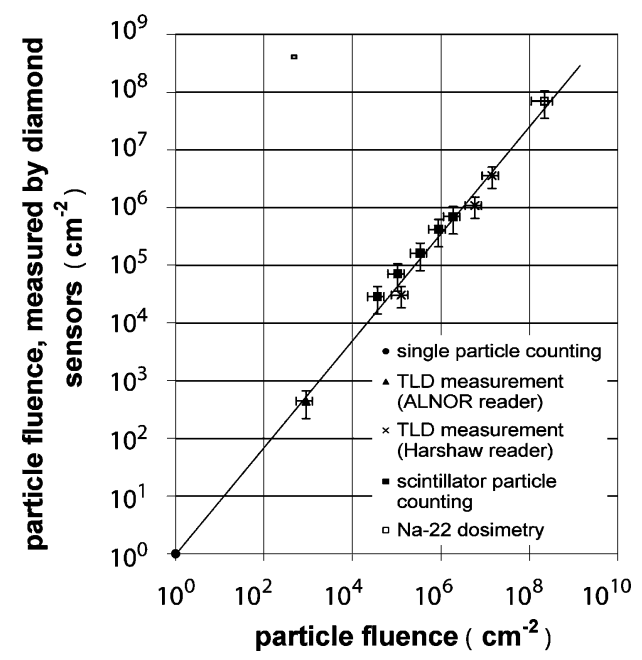

Fig. 7. Diamond sensor response to the particle fluence, as measured by a scintillator hodoscope of $5 \mathrm{~mm} \times 5 \mathrm{~mm}$ cross section, by Na-22 dosimetry in aluminium, thermoluminescence dosimetry, and by particle counting with scintillators).

monitoring normal LHC beam conditions, but also to detect abnormal conditions, up to full scale failures like in the event of an unsynchronized beam abort.

\section{CONCLUSIONS}

CVD diamond is able to withstand intense beams up to a level equivalent to an unsynchronized beam abort within the CMS experiment. The diamond-sensor response to a range of fluences shows a linearity over 8 orders of magnitude, making it suitable for the detection of the onset of beam accidents.

Well defined signals from single particles can be achieved by orienting the sensor parallel to the beam. The need to monitor LHC pilot runs, as well as standard data taking runs implies a need for single particle sensitivity, which in turn sets the placement and orientation of BCM sensors within CMS. Placement 
of BCM sensors parallel to the beam axis, close to the beam pipe, permits sensitivity to single particles, as they occur during normal LHC operation, but allows as well to fulfill monitoring tasks during LHC pilot runs. Finally, the detection of the onset of adverse beam conditions is also possible with this sensor configuration.

Although the development of the system initially was started after discussions related to the CMS experiment, the technology is sufficiently general to find applications elsewhere.

\section{ACKNOWLEDGMENT}

The authors wish to thank Element 6, the CERN RD42 collaboration and its spokespersons, H. Kagan and P. Weilhammer; R. Steerenberg for the development and operation of the fast extraction proton beam; and M. Glaser and F. Ravotti for their assistance with the high intensity beam test and the dosimetry. The authors would also like to thank E. Tsesmelis and S. Roe for their support.

\section{REFERENCES}

[1] DSS Working Group, A Detector Safety System for the LHC Experiments, Functional Requirements Document. CERN-JCOP-2002-012, Apr. 25, 2002.
[2] M. Fahrer, G. Dirkes, F. Hartmann, S. Heier, A. Macpherson, T. Müller, and T. Weiler, "Beam loss induced electrical stress test on CMS silicon strip modules," Nucl. Instrum. Methods Phys. Res. A, vol. A518, pp. 328-330, 2004.

[3] A. Drozhdin, N. V. Mokhov, and M. Huhtinen, "Impact of the LHC beam abort kicker pre-fire on high luminosity insertion and CMS detector performance," in Proc. 1999 Particle Accelerator Conf., New York, pp. 1231-1233.

[4] W. Adam et al., (The RD42 Collaboration), Development of Diamond Tracking Detectors for High Luminosity Experiments at the LHC, Status Report/RD42, Mar. 2002, CERN/LHCC 2002-010, LHCC-RD-001, Nov. 2003.

[5] A. Oh, "Particle Detection With CVD Diamond," DESY-THESIS1999-022, Hamburg, Germany, 1999.

[6] L. S. Pan, S. Han, and D. R. Kania, Diamond: Electronic Properties and Applications. Norwell, MA: Kluwer, 1995.

[7] T. Behnke, A. Oh, A. Wagner, W. Zeuner, A. Bluhm, and C.-P. Klages et al., "Development of diamond films for particle detector applications," Diam. Rel. Mat., vol. 7, pp. 1553-1557, 1998.

[8] M. Huhtinen, The Radiation Environment at the CMS Experiment at the LHC. Geneva, Germany, CERN, HU-SEFT-R-1996-14, 1996.

[9] R. Schmidt, R. W. Assmann, H. Burkhardt, E. Carlier, B. Dehning, and B. Goddard et al., Beam Loss Scenarios and Strategies for Machine Protection at the LHC, LHC Project Report 665, May 2003.

[10] B. Jeanneret and H. Burkhardt, Measurements of the Beam Losses in the LHC Rings, LHC-BLM-ES-0001.00-rev1.1, 28.02, 2003.

[11] O. Brüning, "Mechanisms for beam losses and their time constants," in Proc. LHC Workshop Chamonix IX, Chamonix, France, Jan. 15-19, 1999, pp. 264-269. 